\title{
Reabilitação estética e funcional das bordas incisais dos dentes anteriores com fechamento de diastemas e reanatomização em resina composta: relato de caso clínico
}

\author{
Gabrielle Ribeiro de FREITAS'; Adriana Maria Ribeiro JUNQUEIRA'; \\ Claudia Cristina da Costa TELLES'; Giovana Rodrigues Perin \\ CARNAÚBA ${ }^{1}$; Waldemir Francisco VIEIRA-JUNIOR' \\ 1 - Faculdade São Leopoldo Mandic, Especialização em Dentística, Campinas-SP, Brasil.
}

\section{Resumo}

Atualmente, a Odontologia proporciona através do uso de técnicas diretas em resina composta, a possibilidade de tratamentos conservadores, que são capazes de cumprir com a demanda estética e funcional dos pacientes. Esse relato de caso descreve um tratamento estético e funcional de dentes superiores anteriores visando fechamentos de diastemas e reanatomizações dentais. Paciente, 24 anos, sexo masculino, apresentava desgaste dental leve associado à presença de bruxismo e refluxo gastroesofágico. A escolha de tratamento priorizou a conservação dos tecidos duros através do clareamento dental, uso de restaurações diretas em resina composta nanohíbrida (Harmonize, Kerr) e a instrução ao paciente para estar em constante acompanhamento multiprofissional visando o controle de suas condições médicas e odontológicas. O resultado apresentado foi satisfatório, reproduzindo boa estética, promovendo saúde, função e proteção contra futuros desgastes. Assim, restaurações diretas realizadas em resina composta nanohíbrida utilizando uma matriz BRB constituem uma opção de tratamento viável e satisfatório para tratar casos de fechamentos de diastema e reanatomizações dos dentes anteriores.

PALAVRAS-CHAVE: Resinas compostas; Estética Dentária; Desgaste dos dentes; Refluxo gastroesofágico. 


\section{Introdução}

A busca por tratamentos odontológicos é cada vez maior em virtude das necessidades estéticas dos pacientes, que podem ocorrer devido a alterações diversas, como assimetrias, alterações de cor, posição, forma e/ou tamanho dos dentes ${ }^{1}$. Uma procura comum ocorre devido a insatisfação perante alterações da forma e posição dos elementos dentais, que geram situações de espaçamentos dentais, os chamados diastemas ${ }^{1,2}$. Diastemas podem ser observados em qualquer região da boca, mas quando atingem a região anterior da maxila causam um desconforto estético ao paciente ${ }^{1}$. Em muitos casos, o tratamento ortodôntico pode resolver e melhorar a estética, porém há casos em que é necessária a intervenção de outras especialidades odontológicas, como a Dentística restauradora, para resultar em uma finalização estética e funcional do tratamento $0^{1,3,4}$.

A estética possui um papel muito importante nas relações sociais e na autoestima das pessoas ${ }^{5}$. Entretanto, considerar apenas a estética harmônica do sorriso, para agradar o paciente e melhorar sua qualidade de vida, é uma meta incompleta, pois além de suprir as vontades do indivíduo, devemos buscar a saúde funcional dele 6 . O desgaste dental, por exemplo, é uma condição fisiológica relacionada com a idade, porém pode se tornar um problema funcional quando o desgaste é acelerado por processos como erosão, abrasão e/ou atrição ${ }^{7}$. Diante da condição clínica, o paciente pode se preocupar pela integridade de seus dentes e buscar ajuda odontológica ${ }^{7}$. Com o avanço dessa situação, uma das consequências que podem ocorrer é o desgaste da incisal dos elementos anteriores superiores e inferiores, podendo deixar uma linha de dentina exposta, o que prejudica as funções das guias anteriores. Esses desgastes podem acontecer pelo atrito entre os dentes ou pela combinação da atrição com processos erosivos ${ }^{8}$.

Os processos de atrição e erosão são frequentemente associados ao bruxismo e ao refluxo gastroesofágico ${ }^{9}$ e podem se relacionar 
devido ao impacto do processo erosivo tornar o elemento dental mais susceptível aos desgastes mecânicos, acelerando o proces-

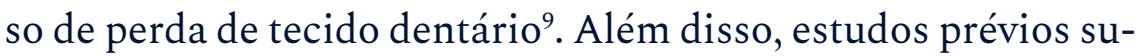
gerem $^{9,10}$ que pode haver uma relação entre bruxismo e refluxo gastroesofágico, ou ainda os associam a outras condições, como a depressão e ansiedade ${ }^{9}$. Essa associação pode ser explicada pelo aumento dos movimentos dos músculos mastigatórios na presença de ácido gástrico $9,11$.

Como opção de tratamento para os casos de desgaste dental uma abordagem minimamente invasiva é necessária ${ }^{7}$. Para isso, o tratamento restaurador direto com resina composta pode ser bem indicado nesses casos, constituindo uma técnica com mínimo ou nenhum desgaste de tecido dental sadio, além de possuir um bom custo-benefício, manutenção simples, e duração satisfatória ${ }^{12}$. O intenso desenvolvimento das técnicas adesivas e restauradoras propicia uma longevidade reparável de dentes com desgastes ${ }^{13}$. Além disso, devido à complexidade dos fatores que contribuem para a instalação e progressão do desgaste dental, se torna imprescindível discutir opções de tratamento que consideram todas as condições envolvidas nesse processo ${ }^{7}$.

O presente estudo é um relato de caso clínico sobre fechamento de diastemas e reanatomizações em dentes desgastados com uso de resina composta direta, visando à recuperação de estética, função e proteção dos dentes envolvidos.

\section{Relato de caso}

O caso clínico foi submetido e aprovado por um Comitê de Ética em Pesquisa (CAAE: 42400221.1.0000.5374). Paciente, 24 anos, sexo masculino, compareceu aos serviços da clínica de pós-graduação em Dentística Restauradora da Faculdade São Leopoldo Mandic, com queixas estética e funcional nos dentes anteriores, além do desejo de retirar as contenções ortodônticas nos arcos superior e inferior. $O$ paciente relatou não estar satisfeito com o formato, a cor, e os desgastes generalizados em seus dentes 
(Figura 1-3). Apresentava história médica de refluxo gastroesofágico e transtorno ansioso depressivo, estando em acompanhamento médico e psicológico, e em uso de Pantoprazol, Citalopram e Domperidona.

Durante a anamnese, o paciente relatou tratamento ortodôntico realizado há aproximadamente 10 anos atrás, com uso de contenção superior e inferior desde então. Além disso, contou que fazia uso de uma placa oclusal rígida (dispositivo miorrelaxante) há três anos devido ao bruxismo durante a noite e relatou ranger os dentes também durante o dia. O paciente complementou que após começar o uso da placa, as dores nos músculos da face haviam cessado e percebeu que os desgastes presentes não

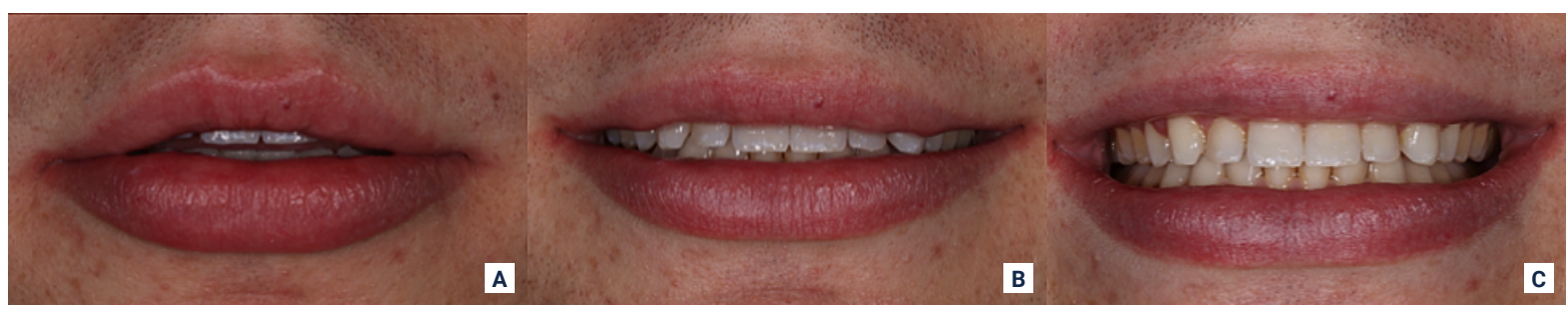

FIGURA 1 - (A) Foto inicial: boca entreaberta. (B) Foto inicial: sorriso leve. (C) Foto inicial: sorriso aberto.

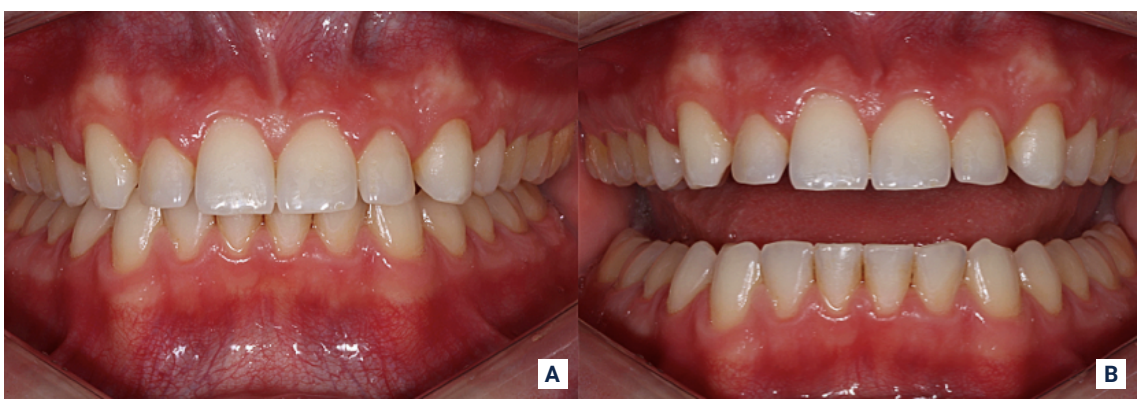

FIGURA 2 • (A) Foto inicial: em máxima intercuspidação. (B) Foto inicial: em desoclusão.

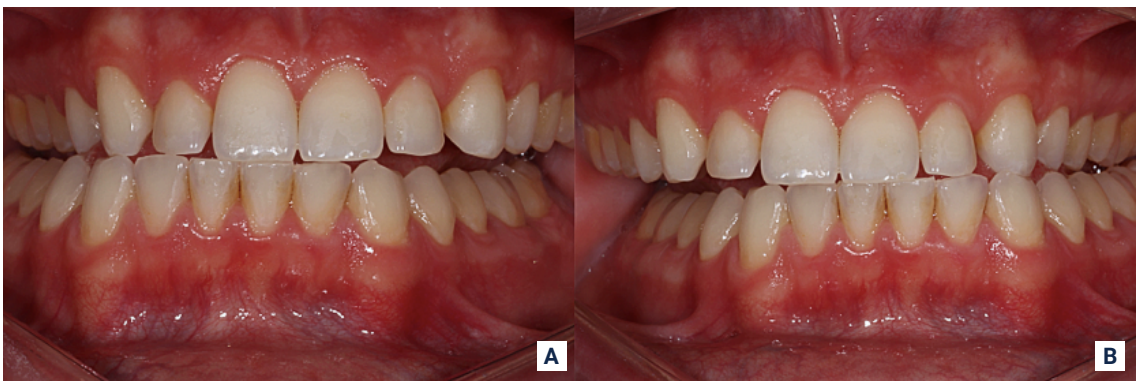

FIGURA 3 • (A) Foto em guia de desoclusão canina direita. (B) Foto em guia de desoclusão canina esquerda. 
evoluíram, entretanto é possível notar uma interferência incisal durante o movimento de lateralidade (Figura 3). No exame clínico, foi possível determinar a cor inicial A3 com auxílio de uma escala de cores (Escala Vita Classical A1-D4, Wilcos, Bad Sackingen, Alemanha), presença de diastemas entre os elementos dentais 13 e 12, 23 e 22, visível desgaste das bordas/pontas dos caninos, facetas de desgaste na incisal dos incisivos inferiores e pequenas fraturas na incisal dos incisivos superiores.

Ao exame radiográfico panorâmico, não foi possível notar alterações significativas, notou-se ausência dos quatro terceiros molares, nenhuma restauração presente em boca. O paciente foi submetido à avaliação periodontal e notou-se a presença pontual de biofilme e cálculo supragengival, que foram removidos profissionalmente. Então, o paciente foi submetido ao procedimento de moldagem com alginato (Hydrogum 5, Zhermack, Badia Polesine, Rovigo, Itália) para confecção de moldeiras de clareamento caseiro. Nesse mesmo dia, foi realizada uma sessão de clareamento de consultório. O primeiro passo foi a instalação do afastador labial e aplicação do gel dessensibilizante à base de nitrato de potássio 3\% e fluoreto de sódio 0,25\% (Dessensibilize UltraEz, Ultradent, South Jordan, Utah, Estados Unidos) em todos os dentes, deixando agir por 15 minutos. Após isso, foi realizada a lavagem abundante do dessensibilizante, secagem dos dentes, e então aplicou-se a barreira gengival fotopolimerizável (Opaldam Green, Ultradent, South Jordan, Utah, Estados Unidos) para proteger o tecido gengival na região cervical de todos os dentes. O gel clareador usado foi à base de peróxido de hidrogénio 40\% (Opalescence Boost PF, Ultradent South Jordan, Utah, Estados Unidos) que foi aplicado de $1^{\circ}$ pré-molar a $1^{\circ}$ pré-molar, na arcada inferior e superior, por 40 minutos.

Ao fim da sessão de clareamento, o gel foi aspirado e os dentes foram lavados em abundância. Após a retirada da barreira gengival e do afastador labial, notou-se uma alteração de cor imediata de A3 para A2 pela escala de cores (Figura 4). O paciente 
apresentou pequenas queixas de sensibilidade apenas nos últimos 5 minutos da sessão. Para realização do clareamento caseiro, foram entregues ao paciente os dispositivos (moldeiras) superior e inferior, três seringas de gel clareador à base de peróxido de carbamida $15 \%$ e nitrato de potássio 0,11\% (Opalescence PF, Ultradent, South Jordan, Utah, Estados Unidos) e foi orientado a usar durante 30 dias por 4 horas diárias. O paciente retornou após 1 mês para avaliação do clareamento, quando foi possível constatar, com uso da escala Vita, que os dentes passaram da cor A3 para A1. Foi orientado que ele continuasse com o clareamento caseiro por mais 14 dias, então outros 14 dias foram aguardados antes da etapa restauradora iniciar.

Assim, inicialmente a contenção ortodôntica superior foi retirada e então se iniciou a etapa restauradora do tratamento. A técnica utilizada para guiar o trabalho foi a matriz BRB, técnica nomeada a partir das iniciais de seus criadores, Bertholdo, Ricci e Barrote. O primeiro passo da técnica foi a confecção da muralha diretamente na boca do paciente com silicone de condensação (Zetalabor. Zhermack, Badia Polesine, Rovigo, Itália). Então, novas proporções dos dentes foram confeccionadas na própria matriz com auxílio de um compasso seco, régua milimetrada,

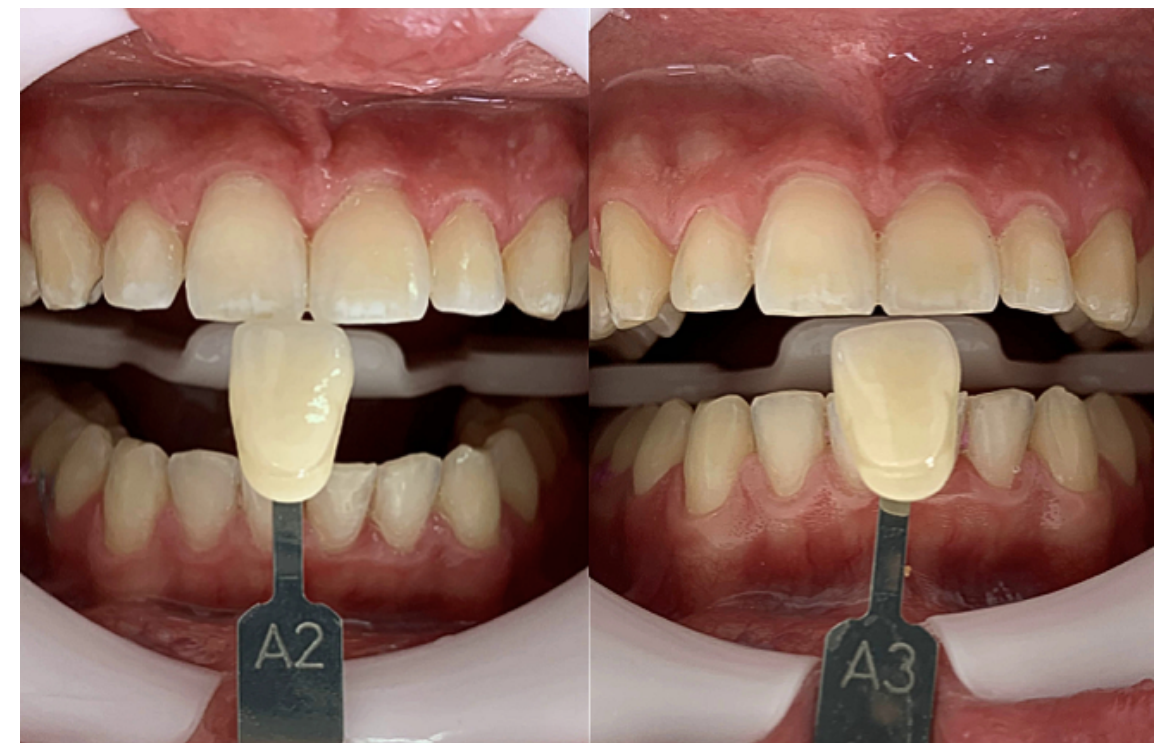

FIGURA 4 - (A) Foto com escala de cor antes da primeira sessão de clareamento em consultório. (B) Foto com escala de cor depois da primeira sessão de clareamento em consultório. 
lápis e uma broca minicut para abrir os novos espaços desenhados (Figura 5). O uso dessa matriz teve como finalidade promover o fechamento dos diastemas presentes entre os elementos 13 e 12, e 23 e 22 , além de promover a reanatomização dos incisivos centrais, laterais e dos caninos.

Com auxílio da escala de cor, foram escolhidas as resinas compostas de cor A2 e A1, as quais foram colocadas sobre a superfície do dente sem condicionamento com ácido prévio e fotoativadas. Assim, foi possível determinar que a cor A1 (Harmonize, Kerr, California, Estados Unidos), se aproximava mais da cor natural dos dentes do paciente. Seguindo o tratamento, foi feito o isolamento absoluto modificado (dos elementos anteriores, e então com uso do ácido fosfórico 35\% (Ultra-Etch, Ultradent, South Jordan, Utah, Estados Unidos) a superfície de esmalte foi condicionada por 30 segundos seguido de lavagem abundante por 30 segundos e secagem da região. Logo após, aplicamos o adesivo (Clearfil SE Bond, Kuraray Noritake Dental, Sakazu, Kurashiki, Okayama, Japão) por toda a superfície condicionada, realizando a fotoativação por 20 segundos com um fotopolimerizador (Valo Cordless, Ultradent, South Jordan, Utah, Estados Unidos). A muralha foi usada para confecção da concha palatina dos elementos. A princípio, foram restaurados os incisivos centrais, seguido dos incisivos laterais, e por fim dos caninos. Para confeccionar a concha palatina e realizar a restauração foi usada a resina nanohíbrida A1E (Harmonize, Kerr, e para dar

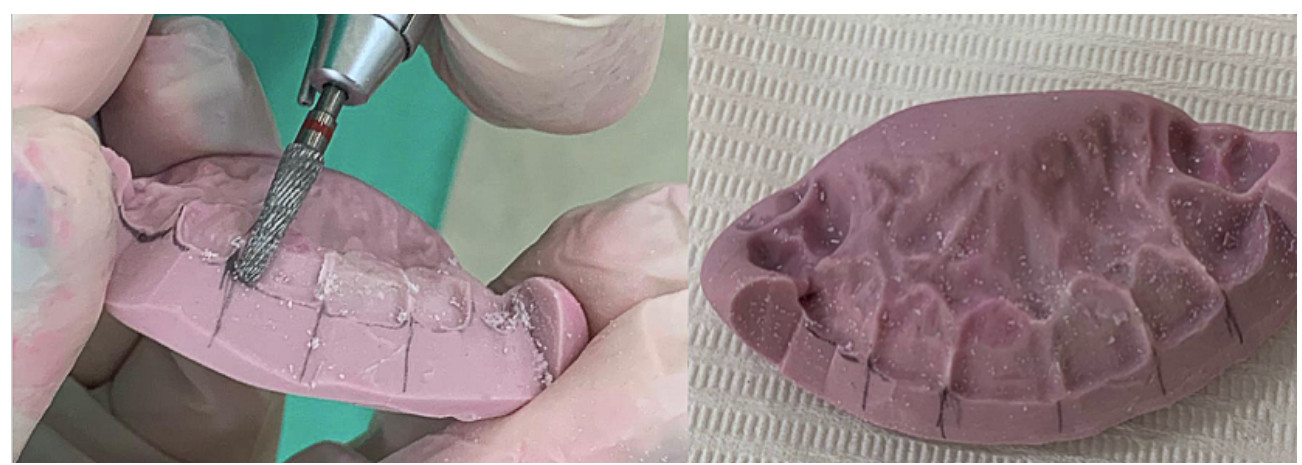

FIGURA 5 • (A) Matriz BRB em confecção. (B) Matriz BRB finalizada. 
os efeitos de translucidez da região incisal foi usado uma resina de efeito (Vit-l-escence IrB, Ultradent, South Jordan, Utah, Estados Unidos).

Após os procedimentos restauradores, o acabamento foi iniciado. Para isso, foi usado o disco abrasivo de cor marrom (Sof-lex Pop On, 3M, Sumaré, São Paulo, Brasil) para retirada mais grosseira dos excessos da resina composta. Para criar a área de luz e sombra dos elementos dentais, foram traçadas as linhas de brilho na superfície vestibular dos dentes para guiar o desgaste com o disco abrasivo amarelo (Sof-lex Pop On, 3M, Sumaré, São Paulo, Brasil) visando delimitar a face vestibular das faces proximais. Finalizada essa etapa, os contatos oclusais foram averiguados e os ajustes necessários foram realizados.

A próxima etapa do acabamento visou à texturização dos dentes, e com auxílio de uma broca diamantada 2200FF (KG Sorensen, Cotia, São Paulo, Brasil) foram feitas microrranhuras na horizontal e vertical. Depois, com as pontas de borracha verde, amarela e branca (Jiffy Polisher Cup, Ultradent, South Jordan, Utah, Estados Unidos), foi feita a suavização desses desgastes até obter mais naturalidade. As proximais também foram refinadas com uso de uma tira de lixa modificada (Soflex, 3M, Sumaré, São Paulo, Brasil), com atenção para não remover os pontos de contato. O polimento foi feito com escovas de carbeto de silício (Occlubrush, Kerr, Orange, California, Estados Unidos) e finalizado com disco de feltro (FPM12CA, DHpro, Paranaguá, Paraná, Brasil) usando uma pasta diamantada (Enamelize, Cosmedent, Friedberg, Alemanha) para dar um maior brilho à resina. Ao final da sessão, moldagem com alginato (Hydrogum 5, Zhermack, Badia Polesine, Rovigo, Itália) e registro de mordida com silicone de condensação (Zetalabor, Zhermack, Badia Polesine, Rovigo, Itália) foram realizados para confecção de uma nova placa miorrelaxante de resina acrílica. 
$\mathrm{Na}$ última sessão, foi entregue ao paciente a placa miorrelaxante acrílica (Figura 6) e foram realizados os devidos ajustes oclusais visando o correto posicionamento da oclusão com o uso da placa, de forma que obtivéssemos contato da placa com todos os dentes. Finalizada a etapa restauradora o paciente foi encaminhado para o ortodontista para avaliação e colocação de nova contenção.

Ao final do tratamento, o paciente se mostrou satisfeito com os resultados (Figura 7 e 8) e assegurou que suas queixas principais foram resolvidas, sem que houvesse desgaste de seus elementos dentais. Houve a recuperação morfológica dos caninos, garantindo ao paciente o restabelecimento funcional das guias anteriores e proteção contra futuros desgastes. $O$ paciente foi orientado a manter o acompanhamento periódico para realização de polimentos, reparos que venham a ser necessários, para que assim o tratamento possa ter uma duração adequada. Também foi orientado a continuar com os acompanhamentos médicos e psicológicos, pois suas condições de saúde poderiam interferir na longevidade e sucesso do tratamento.

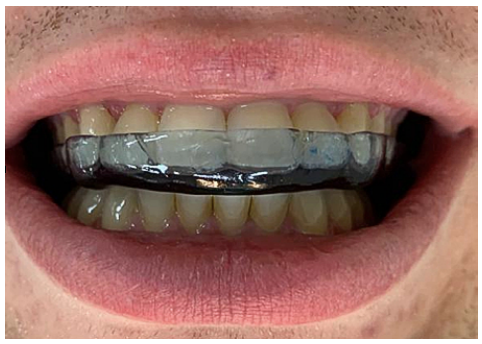

FIGURA 6 - Placa miorrelaxante em acrílico colocada.

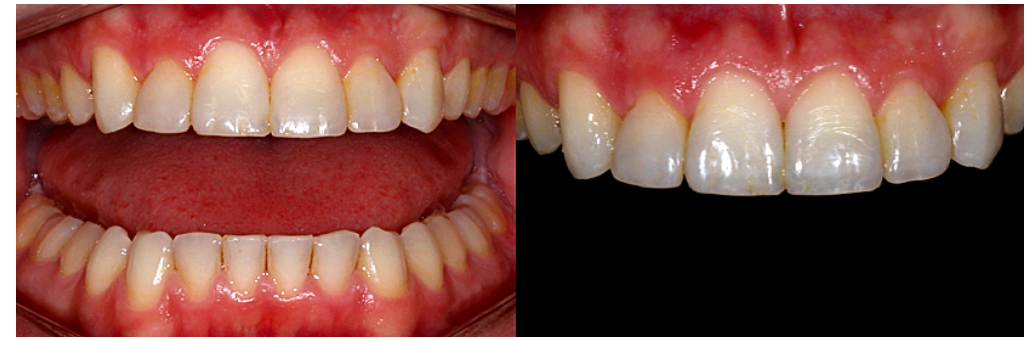

FIGURA 7 · (A) Foto final: em desoclusão. (B) Foto final: dentes superiores anteriores restaurados.

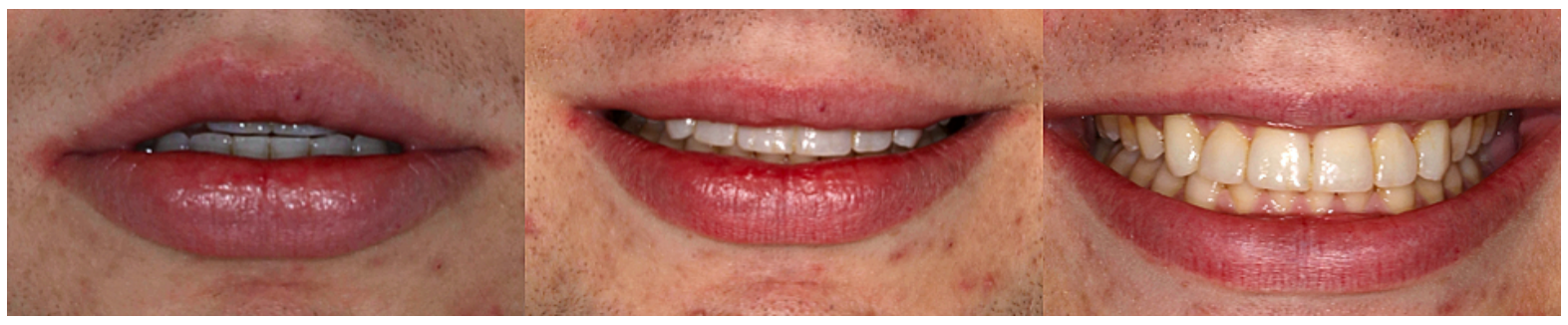

FIGURA 8 - (A) Foto final: boca entreaberta. (B) Foto final: sorriso leve. (C) Foto final: em oclusão. 


\section{Discussão}

É comum as pessoas apresentarem insatisfações estéticas com seus sorrisos devido a alterações de forma, tamanho e cor dos dentes. Para iniciar um tratamento de restauração dental é necessário diagnosticar corretamente a condição patológica associada ao nível de desgaste, há quanto tempo ocorre, identificar se o processo está contido ou em progressão, para assim, ser possível determinar a melhor conduta para o caso ${ }^{14}$. No caso relatado, o paciente, além de ser jovem, se enquadrava em um diagnóstico de desgaste dental inicial ${ }^{15}$. Além disso, visando proteger as estruturas dentais de uma maior perda dentária ${ }^{15}$, o tratamento se baseou na aplicação da técnica restauradora direta em resina composta, já que devido ao seu aspecto conservador quando corretamente indicado e realizado leva à saúde geral dos elementos dentais, e permite que novos tratamentos possam ser viabilizados no futuro, caso sejam necessários ${ }^{16}$.

Além da adequada indicação para o caso em questão, estudos demonstram bons resultados de longevidade ${ }^{17-20}$. O tratamento com resina composta apresenta vantagens, como a possibilidade de realizar pequenos reparos, boa mimetização dos dentes e diminuição do tempo de trabalho ${ }^{1,20,21}$. Entretanto, é importante salientar ao paciente que esse tipo de abordagem necessita de assiduidade nas consultas odontológicas e manutenção do tratamento, visando a realização de profilaxia, pequenos reparos de fraturas, novos acabamentos e repolimentos, visando o sucesso e longevidade do trabalho realizado ${ }^{7,14}$.

Ainda abordando as opções de tratamento para o caso, outra opção que constituía indicação para o caso, seria o uso de técnica indireta com cerâmica ${ }^{15}$. Pensando na situação do paciente, que constituía em perda de tecido dentário em estágio inicial, essa escolha foi descartada, por necessitar de desgaste das estruturas dentais sadias, e a abordagem adotada buscava impedir maiores perdas. Além do mais, a escolha por tratamento indireto com cerâmica não foi feita por se tratar de um desgaste inicial e 
controlado, somada ao fato de o paciente ter intacta sua dimensão vertical ${ }^{7,22}$. Outro fator negativo para o uso de uma técnica indireta era que a região intrassulcular não necessitava de intervenção, sendo necessário apenas acréscimo de material na região de terço médio e incisal dos elementos anteriores superiores, ademais, o caso constituía primeira tentativa de tratamento e controle, apresentando prognóstico favorável ${ }^{7,22}$.

Para o início do tratamento restaurador, foi necessário aguardar 14 dias entre a finalização do clareamento e o dia da realização das restaurações ${ }^{23,24}$. Isso se deve ao fato do gel clareador promover a formação de oxigênio residual na superfície do den$\mathrm{te}^{23}$, o que interfere negativamente nas forças de adesão das restaurações adesivas feitas em dentes recentemente clareados ${ }^{24}$. Além disso, uma restauração em dentes anteriores, pelo apelo estético da região, deve mimetizar textura, variação cromática, translucidez e brilho natural dos dentes, e atender às questões mecânicas com valores aceitáveis de resistência ao desgaste, força, e também promover um bom vedamento das margens da restauração $0^{25}$.

Tendo em vista esses pré-requisitos, uma resina composta nanohíbrida foi escolhida como o material para realizar a restauração direta, já que esse material promove estabilidade de cor, baixa degradação da matriz de resina ${ }^{26,27,}$ e ótimo resultados após acabamento e polimento ${ }^{28,29}$. Isso parece se justificar pela adição de nanopartículas de carga de sílica e vidro na matriz de resina, que preenchem os espaços entre as partículas maiores ${ }^{26,27,30}$, deixando a restauração menos sujeita às ações hidrolíticas, além de proporcionar melhores propriedades mecânicas, como uma resistência ao desgaste satisfatória ${ }^{26,27,30}$. Corroborando com um estudo prévio ${ }^{21}$, o presente caso demonstra que a escolha pela resina nanohíbrida, para casos de fechamento de diastemas e reanatomizações foi capaz de suprir a demanda, apresentando bons resultados estéticos e funcionais. 
Atualmente, várias técnicas para realização de trabalhos restauradores foram descritas, como o uso de coroas manufaturadas, trabalho à mão livre com ou sem ajuda de tiras de matriz, e uso de guias de silicone ${ }^{31}$. Essa última tem como finalidade guiar a restauração proporcionando ao operador maior noção de tamanho e largura dos dentes que serão trabalhados ${ }^{32,33}$. Com a guia de silicone é possível criar a concha palatina, que facilita a confecção do halo incisal, o que dá a restauração final característica mais fidedignas, criando uma camada de esmalte em resina com cor, brilho, translucidez, e opacidade bem próximas ao dente natural ${ }^{31}$. Apesar de o uso da guia de silicone caracterizar uma técnica que facilita o trabalho do operador, ela também aumenta o preço da operação e número de sessões ${ }^{33}$.

Em vista disso, no presente relato de caso, optamos por uma técnica modificada de guia de silicone, a matriz $\mathrm{BRB}^{34}$. Essa técnica consiste em obter a guia de silicone diretamente no paciente, sem intermédio de modelos encerados, para que os novos formatos almejados sejam conseguidos e possam ser confeccionados através de aberturas de espaço na própria guia de silicone ${ }^{34}$. Essa confecção se dá com uso de compasso seco e régua milimetrada, o que permite medir corretamente os espaços que serão abertos, visando à harmonia com os elementos já presentes. Já o desgaste para abertura é realizado com uma broca de tungstênio (minicut) e lâmina de bisturi ${ }^{34}$. Assim como nesse trabalho, um relato de caso realizado anteriormente ${ }^{35}$, concluiu que a técnica possibilita resultados estéticos satisfatórios, com diminuição dos custos, e menor número de sessões clínicas.

No fim do tratamento, o paciente foi instruído quanto à importância de manter sob controle suas condições sistêmicas, tendo em vista que apresentava história médica positiva para refluxo gastroesofágico e transtorno ansioso depressivo, além de ser bruxista em uso de dispositivo miorrelaxante. Isso porque, estudos mostram evidências que relacionam refluxo e bruxismo ${ }^{9-11,36-38}$, em outros há relação que engloba ainda o refluxo, 
bruxismo e transtornos psicológicos ${ }^{37,39}$, sendo que uma condição influencia a outra, desencadeando consequências odontológicas como a instalação de um processo patológico de desgaste denta ${ }^{37}$. Visando a melhora na qualidade de vida, essas condições devem ser tratadas ou controladas ${ }^{40}$. Para quadros de bruxismo é preciso sempre identificar as causas e implementar medidas conservadoras, tentando mediar a condição ${ }^{42}$. Dentre diferentes abordagens terapêuticas para o bruxismo, podem ser usados dispositivos miorrelaxante em acrílico que irão proteger os dentes do desgaste ${ }^{41}$; aplicação de toxina botulínica para reduzir a hiperatividade muscular ${ }^{43-46}$; técnicas de biofeedback como terapia cognitivo comportamental visando a regulação da atividade excessiva do músculo ${ }^{47,48}$; e até mesmo lançar mão do uso de aplicativos que ajudam o paciente a lembrar de desencostar os dentes ${ }^{49}$.

Além disso, o cirurgião-dentista deve realizar o encaminhamento aos profissionais especialistas na área médica para avaliação e tratamento da parte sistêmica, como o refluxo e os transtornos de ansiedade relatados. Assim, o paciente deve manter o acompanhamento multiprofissional, de forma a controlar todas as condições de saúde médica e odontológica, no intuito de um prognóstico mais favorável.

\section{Conclusão}

As restaurações diretas realizadas em resina composta nanohíbrida com auxílio da técnica de matriz BRB constituem uma opção de tratamento viável e satisfatório para tratar casos de fechamentos de diastema e reanatomizações dentais de elementos superiores anteriores em casos de desgaste dental.

\section{Referências}

1 - Almeida L, Maran BM, Andrade GS, Naufel FS, Schmitt VL.

Reabilitação estética de diastemas anterossuperiores com resina composta após abordagem ortodôntica. Clin Lab Res Den. 2019;12-31. 
2- Raju A, Mahendra S, Balamohan S, Gopinath S. Correction of relapsed midline diastema. J Indian Orthod Soc. 2020; 54(4):384-385.

3 - Oquendo A, Brea L, David S, Diastema: correction of excessive spaces in the esthetic zone. Dent Clin North Am. 2011; 55(2):265-281.

4 - Saratti CM, Krejci I, Rocca GT. Multiple diastema closure in periodontally compromised teeth: How to achieve an enamel-like emergence profile. J Prosthet Dent. 2016; 116(5):642-646.

5 - Klages U, Zentner A. Dentofacial aesthetics and quality of life. Seminars in Orthodontics. 2007; 13(2):104-115.

6 - Santos BC, Dantas LF, Silva SC, Lima LHA, Agra DM, Fernandes DC. Odontologia estética e qualidade de vida: revisão integrativa. CBios. 2017; 3(3):91.

7- Loomans B, Opdam N, Attin T, Bartlett D, Edelhoff D, Frankenberger $\mathrm{R}$ et al. Severe tooth wear: european consensus statement on management guidelines. J Adhes Dent. 2017; 19(2):111-119.

8 - Bartlett D, O`Toole S. Tooth wear and aging. Aus Dent J. 2019; 64(suppl. 1): 59-62.

9 - Li Y, Yu F, Niu L, Hu W, Long Y, Tay FR et al. Associations among bruxism, gastroesophageal reflux disease, and tooth wear. J Clin Med. 2018; 7(11):417.

10 - Mengatto CM, Dalberto CS, Scheeren B, Barros SGS. Association between sleep bruxism and gastroesophageal reflux disease. J Prosthet Dent. 2013; 110(5):349-355.

11 - Ohmure H, Oikawa K, Kanematsu K, Saito Y, Yamamoto T, Nagahama $\mathrm{H}$, et al. Influence of experimental esophageal acidification on sleep bruxism: a randomized trial. J Dent Res. 2011; 90(5):665-671.

12 - Ahmed KE, Murbay S. Survival rates of anterior composites in managing tooth wear: systematic review. J Oral Rehabil. 2016; 43(2);145-153.

13 - Mengatto CM, Coelho-de-Souza FH, Souza Júnior OB. Sleep bruxism: challenges and restorative solutions. Clin Cosmet Investig Dent. 2016; 8:71-77.

14 - Meyes IA. Minimum intervation dentistry and the management of tooth wear in general practice. Aus Dent J. 2013; 58(1):60-65.

15 - Mesko ME, Sarkis-Onofre R, Cenci MS, Opdam NJ, Loomans B, Cenci TP. Rehabilitation of severely worn teeth: A systematic review. J Dent. 2016; 48: 9-15.

16 - Mackenzie L, Banerjee A. Minimally invasive direct restorations: a practical guide. Br Dent J. 2017; 223(3):163-171.

17 - Loomans B, Kreulen CM, Hujis-Visser HECE, Sterenborg BAMM, Bronkhorst EM, Huysmans MCDNJM, et al. Clinical performance of full rehabilitations with direct composite in severe tooth wear patients: 3.5 years results. J Dent. 2018; 70:97-103. 
18 - Hamburger JT, Opdam NK, Bronkhorst EM, Kreulen CM, Roeters JJ, Huysmans MC. Clinical performance of direct composite restorations for treatment of severe tooth wear. J Adhes Dent. 2011;13(6):585-593.

19 - Milosevic A, Burnside G. The survival of direct composite restorations in the management of severe tooth wear including attrition and erosion: a prospective 8-year study. J Dent. 2016; 44:13-19.

20 - Lempel E, Lovász BV, Meszarics R, Jeges S, Tóth A, Szalma J. Direct resin composite restorations for fractured maxillary teeth and diastema closure: a 7 years retrospective evaluation of survival and influencing factors. Dent Mater. 2017; 33(4):467-476.

21 - Ergin E, Kutuk ZB, Cakir FY, Gurgan S. Comparison of two different composite resins used for tooth reshaping and diastema closure in a 4-year follow-up. Niger J Clin Pract. 2018; 21(9):1098-1106.

22 - Opdam NJM, Frankenberger R, Magne P. From direct versus indirect towards an integrated restorative concept in the posterior dentition. Oper Dent. 2016; 41(S7):S27-S34.

23 - Mounika A, Mandava J, Roospesh B, Karri G. Clinical evaluation of color change and tooth sensitivity with in-office and home bleaching treatments. Indian J Dent Res. 2018; 29(4):423-427.

24 - Attin T, Hannig C, Wiegand A, Attin R. Effect of bleaching on restorative materials and restorations--a systematic review. Dent Mater. 2004; 20(9): 852-861.

25 - Demirci M, Tuncer S, Sancakli HS, Tekçe N, Baydemir C. Five-year clinical evaluation of a nanofilled and nanohybrid composite in class IV cavities. Oper Dent. 2018; 43(3):261-271.

26 - Akalin TT, Genc G, Ceyhan YK, Bozkurt FO. The effects of mouth rinses on the color stability of sonicfill and a nanohybrid composite. J Istanb Univ Fac Dent. 2016; 50(2):17-23.

27 - Yu B, Lim HN, Lee YK. Influence of nano and micro filler proportions on the optical property stability of experimental dental resin composites. Mater Design. 2010; 31(10):4719-4724.

28 - Soliman HAN, Eikholany NR, Hamama HH, El-Sharkawy FM, Mahmoud SL, Comisi JC. Effect of different polishing systems on the surface roughness and gloss of novel nanohybrid resin composites. Eur J Dent. 2020; 15(2):10-27.

29 - Nashaat MM, Kola MZ, Algahtani HH, Algahtani MD, Alghmlas AS. Evaluation of surface roughness of different direct resin based composites. J Int Soc Prevent Communit Dent. 2017; 7(3):104-109.

30 - Velo MMAC, Coelho LVBF, Bating RT, Amaral FLB, França FMG. Longevity of restorations in direct composite resin: literature review. Rev Gaúch Odontol. 2016; 64(3):320-326.

31 - Denehy GE. Simplifying the class IV lingual matrix. J Esthet Restor Dent. 2006; 17(5): 312-319. 
32 - Detogni AC, Plana EA, Gadonski AP, Camilotti V. Reanatomização dental pela técnica direta com guias de silicone: relato de caso clínico. Clin Lab Res Den. 2020;8(30):1-9.

33 - Silva GR, Waechter DM, Martins LRM, Barreto BCF, Soares CJ. Técnicas restauradoras para fraturas coronárias de dentes anteriores traumatizados. UNOPAR Cient Ciênc Biol Saúde. 2012; 14(4): 251-256.

34 - Bertholdo G, Barrote LGA, Ricci WA. Matriz Bertholdo/Ricci/ Barrote (BRB): uma simplificação da técnica para obtenção de guia de estratificação com compósitos. Clin Int J Braz Dent. 2014; 10(2): 204-213.

35 - Pombo SQR, Espíndola-Castro LF, Silva CHV, Salvador DPT, Monteiro GQM, Menezes Filho PF. Reanatomização dental com resina composta utilizando matriz BRB: relato de caso. Rev Ciên Saúde. 2020; 5(2): 1-7.

36 - Wetselaar P, Manfredini D, Ahlberg J, Johansson A, Aarab G, Papagianni CE et al. Associations between tooth wear and dental sleep disorders: a narrative overview. J Oral Rehabil. 2019; 46(8): 765-775.

37 - Li Y, Yu F, Niu L, Long Y, Tay FR, Chen J. Association between bruxism and symptomatic gastroesophageal reflux disease: a casecontrol study. J Dent. 2018; 77:51-58.

38 - Miyawaki S, Tanimoto Y, Araki Y, Katayama A, Imai M, TakanoYamamoto T. Relationships among nocturnal jaw muscle activities, decreased esophageal pH, and sleep positions. Am J Orthod Dentofac Orthop. 2004; 126(5):615-619.

39 - You ZH, Perng CL, Hu LY, Lu T, Chen PM, Yang AC, et al. Risk of psychiatric disorders following gastroesophageal reflux disease: a nationwide population-based cohort study. Eur J Intern Med. 2015; 26(7): 534-539.

40 - Iwakiri K, Kinoshita Y, Habu Y, Oshima T, Manabe N, Fujiwara Y, et al. Evidence-based clinical practice guidelines for gastroesophageal reflux disease 2015. J Gastroenterol. 2016; 51(8):751-767.

41 - Gholampour S, Gholampour H, Khanmohammadi H. Finite element analysis of oclusal splint therapy in patients with bruxism. BMC Oral Health. 2019;19(1): 205.

42 - Manfredini D, Colonna A, Bracci A, Lobbezoo F. Bruxism: a summary of current knowledge on aetiology, assessment and management. Oral Surg. 2019; 13(4):358-370.

43 - Fernández-Nuñez T, Amghar-Maach S, Gay-Escoda C. Efficacy of botulinum toxin in the treatment of bruxism: Systematic review. Med Oral Patol Oral Cir Bucal. 2019; 24(4):e416-e424.

44 - Patel J, Cardoso JA, Mehta S. A systematic review of botulinum toxin in the management of patients with temporomandibular disorders and bruxism. Br Dent J. 2019; 226(9): 667-672. 
45 - Yurttutan ME, Sancak KT, Tüzüner AM. Wich treatment is effective for bruxism: occlusal splints or botulinum toxin? J Oral Maxillofac Surg. 2019; 77(12): 2431-2438.

46 - Kumar A, Spivakovsky S. Bruxism: is botulinum toxin an effective treatment? Evid Based Dent. 2018;19:59.

47 - Sato M, Iizuka T, Watanabe A, Iwase N, Otsuka H, Terada N, et al. Eletromyogram biofeedback training for daytime clenching and its effect on sleep bruxism. J Oral Rehab. 2014; 42(2):83-89.

48 - Jokubauskas L, Baltrusaitye A. Efficacy of biofeedback therapy on sleep bruxism: A systematic review and meta-analysis. J Oral Rehab. 2018; 45(6): 485-495.

49 - Zani A, Lobbezoo F, Bracci A, Ahlberg J, Manfredini D. Ecological momentary assessment and intervention principles for the study of awake bruxism behaviors, part 1: general principles and preliminary data on healthy young Italian adults. Front Neurol. 2019;10:169. 


\title{
Esthetic and functional rehabilitation of the incisal edges of anterior teeth with diastema closure and reanatomization with composite resin: a clinical case report
}

\begin{abstract}
Currently, Dentistry offers the possibility of conservative treatments using direct techniques with composite resin, which are able to achieve the esthetic and functional demands of patients. This case report describes an esthetic and functional treatment of the superior anterior teeth, which aimed to close diastemas restoring the teeth. The patient, 24 years old, male gender, showed teeth wear associated with the presence of bruxism and gatroesophageal reflux. The choice of treatment prioritized the conservation of hard tissue through tooth bleaching and use of direct restorations with nanohybrid resin composite (Harmonize, Kerr), as well as instructing the patient to regularly engage in multiprofessional follow-up in order to monitor their medical and odontological condition. The shown result was satisfactory, reproducing good aesthetic, promoting health, function, and protection against future wear. Thus, direct restorations made of nanohybrid resin composite utilizing the BRB matrix make up a treatment option that is feasible and satisfactory to treat cases of diastema closure and reanatomization of the anterior teeth.
\end{abstract}

Keywords: Composite resins; Aesthetic; Tooth wear; Gastroesophageal reflux disease.

\section{Como citar este artigo}

Freitas GR, Junqueira AMR, Telles CCC, Carnaúba GRP, Vieira-Junior WF. Reabilitação estética e funcional das bordas incisais dos dentes anteriores com fechamento de diastemas e reanatomização em resina composta: relato de caso clínico. Rev Odontol Bras Central 2021; 30(89): 339-356. DOI: 10.36065/robrac.v30i89.1537 